\title{
Background familiar e desempenho escolar: uma abordagem com variáveis binárias a partir dos resultados do Saresp
}

\author{
Camila Fernanda Bassetto*
}

No presente estudo investiga-se o impacto de fatores externos à escola sobre a escolarização do indivíduo. Renda e escolaridade materna são consideradas para medir o efeito de aspectos familiares no desempenho em matemática do aluno. Para tanto, foram utilizados dados referentes à nota em matemática dos alunos da $3^{3}$ a série do ensino médio e as respostas dos pais ao questionário socioeconômico, aplicado pelo Sistema de Avaliação do Rendimento Escolar do Estado de São Paulo (Saresp) na edição de 2013. São propostos modelos com variáveis binárias e obtidas estimativas para os parâmetros por meio do software SPSS. Os resultados mostram que rendas mais altas proporcionam melhores desempenhos educacionais, porém há restrições. O nível de escolaridade materna atua positivamente sobre o desempenho do aluno, mas está limitado ao nível adequado de proficiência.

Palavras-chave: Proficiência em matemática. Renda familiar. Escolaridade materna. Saresp. Variáveis binárias.

\footnotetext{
* Faculdade de Ciências e Letras (FCL), Universidade Estadual Paulista “Júlio de Mesquita Filho" (Unesp), Araraquara-SP, Brasil (camila.bassetto@unesp.br; http://orcid.org/0000-0002-2513-7455).
} 


\section{Introdução}

A avaliação educacional se faz presente em várias instâncias da ação humana e caracteriza-se como ferramenta útil para melhorar o sistema educacional, pois disponibiliza informações que possibilitam aos educadores distinguir as práticas que promovem os resultados desejados daquelas menos eficazes. Concomitantemente, a avaliação deve proporcionar um monitoramento contínuo do sistema educacional, buscando detectar os efeitos positivos e negativos das políticas adotadas (SOARES; COLLARES, 2006).

No Brasil, é perceptível o avanço empreendido na avaliação educacional, seja por intermédio dos recursos metodológicos de que se dispõe, seja por meio dos sistemas de avaliações atualmente operacionalizados, tais como o Sistema Nacional de Avaliação da Educação Básica (Saeb), o Sistema de Avaliação do Rendimento Escolar do Estado de São Paulo (Saresp), o Exame Nacional do Ensino Médio (Enem) e o Exame Nacional de Desempenho dos Estudantes (Enade), entre outros. Os resultados dessas avaliações têm revelado novas faces das desigualdades educacionais no país, com um quadro crítico da educação formal em relação à qualidade do ensino, ao rendimento escolar do indivíduo e às disparidades de desempenho escolar entre os estratos regionais e sociais. De acordo com o Relatório Pedagógico do Saresp de 2013 (SÃO PAULO, 2013), aproximadamente $55 \%$ dos alunos da 3 a série do ensino médio da rede estadual encontram-se no mais baixo nível de proficiência em matemática, isto é, apresentam habilidades limitadas para solucionar, ou são incapazes de resolver, questões propostas para a série em que se encontram.

Na avaliação educacional, o desempenho do aluno é considerado a variável indicadora da eficácia deste sistema e é coletado juntamente com informações acerca do contexto escolar e social do indivíduo, características de interesse de diversos estudos (FERRÃO et al., 2001; ALVES; SOARES, 2008; FRANCO; MENEZES-FILHO, 2012). Ao longo da última década, estudos têm revelado, por meio de evidências empíricas, que o desempenho escolar dos alunos depende não só das habilidades próprias, mas também de fatores que envolvem características sociais, econômicas, culturais e familiares (SOARES; MENDONÇA, 2003; JESUS; LAROS, 2004; ALVES; SOARES, 2008). Tais autores argumentam que o desempenho do aluno deve ser contextualizado nas avaliações educacionais, uma vez que as desigualdades sociais têm implicações diretas sobre a educação.

Embora a literatura evidencie um consenso entre autores que afirmam que a renda familiar, como característica extraescolar, impacta diretamente o desempenho escolar do aluno, como os estudos citados previamente, há de se indagar se é sempre válido afirmar que rendas mais altas geram melhores desempenhos, ou seja, se melhores condições financeiras estão associadas às médias mais altas em matemática. Indagações também se fazem presentes quanto à influência da escolaridade materna sobre o desempenho educacional do filho. Alunos cujas mães possuem níveis mais altos de instrução alcançam melhores desempenhos escolares? 
Buscando respostas para as questões levantadas e com o propósito de contribuir com a literatura que investiga os fatores que atuam sobre o desempenho escolar do indivíduo, o presente estudo tem por objetivo medir o impacto da renda familiar e da escolaridade da mãe sobre o desempenho em matemática do estudante da 3 á série do ensino médio matriculado na rede pública no estado de São Paulo. A metodologia proposta para alcançar tal objetivo é baseada em modelos lineares com variáveis binárias e com a utilização de dados referentes às notas em matemática alcançadas no Saresp de 2013. São utilizadas também as respostas dos pais dos alunos às questões sobre renda familiar e nível de instrução materna, coletadas por esta avaliação mediante aplicação de um questionário socioeconômico. Os efeitos destas características são medidos por meio de estimativas para os parâmetros dos modelos propostos, as quais são obtidas via software SPSS.

A seguir é feita uma breve revisão de literatura sobre estudos dedicados à identificação e compreensão de fatores, internos e externos à escola, que atuam sobre o desempenho educacional do aluno. Posteriormente, são descritos os níveis de proficiência em matemática registrados pelo Saresp e as questões sobre renda familiar e escolaridade da mãe. Também é realizada a distribuição dos alunos, por nível de proficiência, conforme as faixas de renda e os níveis de instrução materno, identificados em 2013. São apresentados os modelos propostos e a categorização das variáveis qualitativas em binárias, bem como discutidos e interpretados os resultados obtidos a partir da análise empírica. Por fim, faz-se uma conclusão da presente pesquisa.

\section{Renda familiar e escolaridade da mãe: impacto no desempenho escolar}

Estudos realizados nas últimas décadas mostram que o interesse pela investigação dos fatores que interferem no desempenho escolar do aluno tem crescido nos âmbitos nacional e internacional (SOARES; MENDONÇA, 2003; SOARES; COLLARES, 2006; FRANCO; MENEZES-FILHO, 2012). No cenário internacional, tais fatores são estudados há mais de cinco décadas e tiveram, como marco inicial, o chamado Relatório Coleman, no qual foram analisadas as possíveis causas para as diferenças de desempenho entre as escolas norte-americanas, impactando fortemente as políticas públicas nos Estados Unidos e da Sociologia da Educação de forma geral (COLEMAN et al., 1966). Os resultados do Relatório Coleman levaram à conclusão de que a maior parte das desigualdades entre os alunos tem origem nas famílias e no contexto social das escolas. Isso deu início a um debate sobre quais fatores extra e intraescolares, de fato, impactam o desempenho do indivíduo (JESUS; LAROS, 2004; ALVES; SOARES, 2008; PALERMO; SILVA; NOVELLINO, 2014). Nesse sentido, o principal interesse deixou de ser a comparação entre as escolas, conforme o rendimento de seus alunos, e passou a ser a identificação de características de naturezas humana, social, cultural, ética, metodológica e instrumental, que podem atuar sobre o desempenho escolar do indivíduo. 
A determinante para o aspecto socioeconômico está ligada diretamente à renda dos pais e, consequentemente, ao nível educacional e cultural dos mesmos. Dessa forma, os recursos financeiros que estão à disposição dos pais se convertem em investimento educacional para os filhos, considerando que um menor nível educacional proporciona um menor nível socioeconômico em um longo prazo. Ferrão et al. (2001), Barbosa e Fernandes (2001) e Soares e Mendonça (2003) desenvolveram pesquisas para identificar os fatores associados ao desempenho escolar do aluno e à eficiência do sistema educacional. Sob o pressuposto de que o conhecimento prévio do aluno à entrada na escola e seu nível socioeconômico são fortemente correlacionados, os autores concluíram que existe relação positiva entre a proficiência do aluno e seu nível socioeconômico. Este resultado confirma o fato amplamente conhecido de que o desempenho acadêmico do aluno é fortemente influenciado pela envolvente social, cultural e econômica.

Utilizando dados do Saeb de 2001, Soares e Mendonça (2003) e Jesus e Laros (2004) verificaram que alunos com melhores condições socioeconômicas alcançaram melhores desempenhos quando comparados aos demais alunos. Os resultados do estudo de Alves e Soares (2008) corroboraram as conclusões dos trabalhos previamente citados. De acordo com os autores, estudos com dados de avaliação dos sistemas de ensino conduzidos no país mostram que a maior parte da variação nos resultados escolares pode ser explicada por fatores extraescolares associados, principalmente, à origem social dos alunos.

Considerando a proporção de mães com ensino superior para indicar um elevado status socioeconômico, Franco e Menezes-Filho (2012) constataram que alunos pertencentes a famílias com maior poder aquisitivo exibiram níveis de proficiência mais altos, evidenciando elevada estratificação social no sistema educacional brasileiro. Para a proporção de mães com ensino médio, embora não tão acentuada, os resultados mostraram uma relação positiva com o desempenho médio da escola. Soares e Collares (2006) destacaram a possibilidade de perceber que os alunos que possuem estrutura familiar com vínculos saudáveis de relacionamento alcançam melhores desempenhos, devido à abertura para conversar com os pais sobre a vida escolar e também aos insumos culturais que recebem. Barros e Mendonça (1997) mostraram que a relação entre a escolaridade dos pais e o desempenho do aluno é mais estreita do que a relação entre renda e desempenho. Uma das principais justificativas para isso está no fato de a renda ser variável e o conhecimento que os pais adquiriram ao longo da vida é permanente, além do que a escolaridade dos pais é o fator mais relevante para a constituição da renda.

Embora o pai exerça papel significativo na vida do filho, no presente estudo foram considerados somente dados associados à mãe, apoiando-se nos estudos de Rios-Neto et al. (2002) e Riani e Rios-Neto (2008), os quais observaram que a mãe exerce papel determinante no desempenho educacional dos filhos. Com o propósito de contribuir com questões referentes à compreensão dos fatores externos à escola, que atuam sobre a escolarização do indivíduo, na seção seguinte apresentam-se dados sobre a renda familiar e 
a escolaridade materna para os alunos classificados nos diferentes níveis de proficiência em matemática na edição do Saresp de 2013.

\section{Distribuição dos alunos nos níveis de proficiência conforme renda familiar e escolaridade materna}

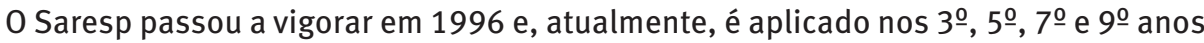
do ensino fundamental e na $3^{a}$ série do ensino médio. 0 resultado alcançado pelo aluno na avaliação descreve o que ele é capaz de fazer em relação às habilidades e competências avaliadas, conforme a Matriz de Referência para Avaliação de Matemática do Saresp. ${ }^{1}$ A partir das expectativas de aprendizagem quanto a conteúdo, competências e habilidades estabelecidos para cada ano/série da disciplina no Currículo do Estado de São Paulo, os pontos da escala do Saresp são agrupados em quatro níveis: abaixo do básico (AB); básico (BA); adequado (AD); e avançado (AV), como mostrado no Quadro 1.

Para alcançar o objetivo proposto no presente estudo, foram utilizadas informações coletadas durante a realização do Saresp em 2013, referentes às notas em matemática dos alunos da 3a série do $\mathrm{EM}^{2}$ e às respostas sobre renda familiar e escolaridade materna, contidas no questionário socioeconômico. ${ }^{3}$

\section{QUADRO 1}

Níveis de proficiência em matemática para a 3ํㅗㄹ série do ensino médio, intervalos de pontuação, classificação e descrição, segundo o Saresp

2013

\begin{tabular}{|llll|}
\hline $\begin{array}{l}\text { Níveis de } \\
\text { proficiência }\end{array}$ & $\begin{array}{l}\text { Intervalos de } \\
\text { pontuação }\end{array}$ & Classificação & Descrição \\
\hline $\begin{array}{l}\text { Abaixo do } \\
\text { básico (AB) }\end{array}$ & Menor que 275 & Insuficiente & $\begin{array}{l}\text { Os alunos demonstram domínio insuficiente dos conteúdos, } \\
\text { competências e habilidades desejáveis para o ano/série em } \\
\text { que se encontram. }\end{array}$ \\
\hline Básico (BA) & $\begin{array}{l}\text { Igual ou acima } \\
\text { de } 275 \text { e abaixo } \\
\text { de } 350\end{array}$ & Suficiente & $\begin{array}{l}\text { Os alunos demonstram domínio mínimo dos conteúdos, } \\
\text { competências e habilidades, mas possuem estruturas } \\
\text { necessárias para interagir com a proposta curricular no ano/ } \\
\text { série subsequente. }\end{array}$ \\
\hline $\begin{array}{l}\text { Adequado } \\
\text { (AD) }\end{array}$ & $\begin{array}{l}\text { Igual ou acima } \\
\text { de } 350 \text { e abaixo } \\
\text { de } 400\end{array}$ & Suficiente & $\begin{array}{l}\text { Os alunos demonstram domínio pleno do conteúdo, } \\
\text { competências e habilidades desejáveis para o ano/série em } \\
\text { que se encontram. }\end{array}$ \\
\hline $\begin{array}{l}\text { Avançado } \\
\text { (AV) }\end{array}$ & $\begin{array}{l}\text { Igual ou acima } \\
\text { de } 400\end{array}$ & Avançado & $\begin{array}{l}\text { Os alunos demonstram conhecimentos e domínio dos } \\
\text { conteúdos, competências e habilidades acima do requerido } \\
\text { no ano/série em que se encontram. }\end{array}$ \\
\hline
\end{tabular}

Fonte: Secretaria da Educação do Estado de São Paulo. Relatório Pedagógico do Saresp 2013.

\footnotetext{
${ }_{1}^{1}$ A Matriz de Referência para Avaliação de Matemática do Enisno Médio pode ser vista em São Paulo (2013).

${ }^{2} 0$ interesse na utilização de uma amostra composta somente por microdados associados à $3^{2}$ série do ensino médio deve-se ao fato de tal série contemplar um conteúdo de matemática cujas bases se fazem presentes no ensino fundamental. Concomitantemente, a quantidade de informações considerando apenas a 3aㅗ série do ensino médio, sem inserir dados do ensino fundamental, proporcionou uma amostra de tamanho significativo para a realização da presente pesquisa. Em futuras investigações, pretende-se incluir dados de alunos matriculados no ensino fundamental.

${ }^{3}$ A escolha do ano 2013 deve-se ao fato de que é a última edição do Saresp a aplicar o questionário socioeconômico aos pais dos alunos participantes, por meio do qual é possível conhecer a renda familiar e o nível de instrução materno, variáveis de interesse nesta pesquisa. Os dados provenientes das demais edições do Saresp correspondem somente ao desempenho dos alunos nas provas.
} 
Tanto o questionário socioeconômico, aplicado aos pais dos alunos, quanto o questionário aplicado às escolas participantes, o qual é respondido por diretores, coordenadores e professores, integram parte fundamental do Saresp, uma vez que proporcionam, aos avaliadores, conhecimento sobre aspectos familiares dos alunos e sobre as escolas, no que se refere à estrutura física, existência de laboratórios, entre outras. Tais questionários são previamente enviados às escolas, que os distribuem aos alunos inscritos na avaliação para que levem até seus pais e o entreguem respondidos no dia da realização da prova. Os microdados e, consequentemente, a amostra elaborada abarcam informações de todos os municípios do estado de São Paulo. Os dados coletados pelo Saresp são organizados e analisados pela Secretaria Estadual de Educação de São Paulo (SEE/SP), especificamente pela Coordenadoria de Informação, Monitoramento e Avaliação Educacional (Cima). ${ }^{4}$

Os níveis de escolaridade das mães dos alunos, disponíveis no questionário socioeconômico, são apresentados no Quadro 2.

\section{QUADRO 2}

Níveis de escolaridade das mães dos alunos, segundo questionário socioeconômico do Saresp 2013
(A) Não estudou
(B) Ensino fundamental (1a a 4a série) incompleto
(C) Ensino fundamental (1 1 a a $\underline{a}$ série) completo

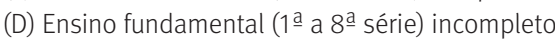
(E) Ensino fundamental ( $1 \stackrel{a}{a}$ a $8^{\underline{a}}$ série) completo
(F) Ensino médio (antigo 2ํo grau) incompleto

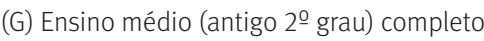
$(\mathrm{H})$ Ensino superior (faculdade) incompleto
(I) Ensino superior (faculdade) completo
(J) Não sabe

Fonte: Saresp 2013 - Questionário socioeconômico.

Nesta pesquisa, buscou-se investigar também o impacto da renda familiar sobre o desempenho escolar do aluno matriculado na 3ạ série do ensino médio da rede pública do estado de São Paulo. A partir dos estudos de Soares e Mendonça (2003) e Jesus e Laros (2004), é possível observar que o nível socioeconômico do aluno pode ser medido por diversos fatores, tais como a escolaridade dos pais, a presença ou ausência de itens específicos em casa (tal como condições sanitárias, geladeira e automóvel) e outros determinantes ligados diretamente ao aspecto socioeconômico. Embora a renda familiar varie durante a vida escolar do aluno, no presente estudo a condição socioeconômica foi classificada conforme a renda familiar, uma vez que tal informação é disponibilizada no questionário socioeconômico do Saresp, como mostra o Quadro 3.

\footnotetext{
${ }^{4}$ Os microdados do Saresp não são públicos e foram disponibilizados após um termo de responsabilidade ter sido firmado entre a Cima e a parte interessada na realização desta pesquisa.
} 


\section{QUADRO 3}

Níveis de renda familiar dos alunos, segundo questionário socioeconômico do Saresp 2013
(A) Até $R \$ 850$
(B) De $R \$ 851$ a $R \$ 1.275$
(C) De $R \$ 1.276$ a $R \$ 2.125$
(D) $\mathrm{De} R \$ 2.126$ a $\mathrm{R} \$ 4.250$
(E) Mais de $R \$ 4.250$
(F) Nenhuma renda
(G) Não sabe/não quer responder

Fonte: Saresp 2013 - Questionário socioeconômico.

A alternativa (A) mostrada no Quadro 3 sugere uma renda familiar próxima de um salário mínimo, a alternativa (B) indica uma renda familiar próxima de até dois salários mínimos, a alternativa (C) abarca rendas familiares entre dois e três salários mínimos, aproximadamente. Rendas familiares mais altas são indicadas nas alternativas (D) e (E), com quantidades entre três e seis salários mínimos na alternativa (D) e superiores a seis na alternativa (E). ${ }^{5}$

No processo de composição da amostra e organização dos dados, as respostas associadas à alternativa (J), apresentada no Quadro 2, e às alternativas (F) e (G), citadas no Quadro 3, foram descartadas, pois não possibilitam qualquer conhecimento sobre a escolaridade da mãe e a renda familiar do aluno. Assim, de um total de 234.963 observações, foram excluídas 39.910 associadas a essas três alternativas, resultando em uma amostra de 195.053 alunos.

Considerando o nível de instrução da mãe, a Tabela 1 apresenta a distribuição dos alunos da amostra, em cada nível de proficiência em matemática, avaliada pelo Saresp 2013.

TABELA 1

Distribuição dos alunos da 3aㅗ série do ensino médio da amostra, por níveis de proficiência em matemática, segundo escolaridade da mãe

Estado de São Paulo - 2013

Em porcentagem

\begin{tabular}{lcccc}
\hline \multirow{2}{*}{ Escolaridade da mãe } & \multicolumn{3}{c}{ Níveis de proficiência em matemática } \\
\cline { 2 - 5 } & $\begin{array}{c}\text { Abaixo do } \\
\text { básico (AB) }\end{array}$ & Básico (BA) & Adequado (AD) & Avançado (AV) \\
\hline Não estudou & 3,2 & 1,9 & 1,1 & 0,6 \\
Ensino fundamental incompleto & 29,3 & 23,9 & 16,5 & 13,8 \\
Ensino fundamental completo & 24,7 & 21,4 & 16,4 & 14,7 \\
Ensino médio incompleto & 7,3 & 7,8 & 7,7 & 7,1 \\
Ensino médio completo & 26,6 & 31,9 & 35,0 & 32,9 \\
Ensino superior incompleto & 3,3 & 4,4 & 6,1 & 5,5 \\
Ensino superior completo & 5,4 & 8,7 & 17,2 & 25,2 \\
Número de obs. & $\mathbf{9 2 . 9 3 3}$ & $\mathbf{8 7 . 2 9 3}$ & $\mathbf{1 3 . 9 2 7}$ & $\mathbf{9 0 0}$ \\
\hline
\end{tabular}

Fonte: Saresp 2013.

Os dados da Tabela 1 indicam que, independentemente do nível de proficiência em matemática do aluno, os menores percentuais estão associados aos alunos cujas mães

\footnotetext{
${ }^{5}$ Em 2013, o salário mínimo vigente era de R\$ 678.
} 
declararam não terem estudado. No entanto, diferentemente dos demais, para o nível AB, tal percentual está bastante próximo daquele correspondente aos alunos com mães que possuem ensino superior incompleto, com uma diferença de apenas $0,1 \%$, o que corresponde a 93 alunos, aproximadamente.

A Tabela 1 permite verificar que, à medida que o desempenho do aluno melhora, atingindo níveis mais altos de proficiência em matemática, conforme estabelecidos pelo Saresp, decrescem os percentuais daqueles com mães que possuem ensino fundamental incompleto e completo. Por outro lado, entre as mães com ensino superior completo, aumentam os percentuais de alunos conforme avança o nível de proficiência, de tal forma que, no nível $A V$ o percentual é quase cinco vezes maior quando comparado ao nível $A B$. Padrão similar é observado no ensino superior incompleto, mas com um percentual maior para os alunos com nível AD de proficiência.

Os maiores percentuais de alunos classificados nos níveis básico, adequado e avançado de proficiência correspondem a mães com ensino médio completo. Para os alunos pertencentes ao nível abaixo do básico, a maioria possui mães com ensino fundamental incompleto.

Entre as mães com ensino médio completo, verificam-se os maiores percentuais de alunos para todos os níveis de proficiência em matemática, exibindo maior valor no nível $A D$, com 35\% do total. Observa-se, também, que, entre as mães com ensino médio incompleto, o menor percentual de alunos encontra-se no nível AV de proficiência, o qual contempla o mais alto percentual de alunos cujas mães possuem ensino superior completo.

Os dados da Tabela 1, ao indicarem que os menores percentuais de alunos com nível AV de proficiência estão entre as mães que não estudaram, que possuem ensino fundamental completo e incompleto e ensino médio incompleto, enquanto os maiores percentuais, para o mesmo nível de proficiência, são observados para as mães com nível de instrução superior ao ensino médio completo, evidenciam um padrão no comportamento do desempenho educacional do aluno, conforme o nível de escolaridade da mãe. Os resultados identificados a partir da amostra considerada nesta pesquisa sugerem que o nível de proficiência dos alunos em matemática aumenta à medida que os anos de estudo da mãe também avançam. Nesse sentido, tais valores indicam a existência de uma relação positiva entre proficiência em matemática e escolaridade materna, ou seja, filhos de mães mais escolarizadas possuem melhores desempenhos em matemática, medidos pelos níveis de proficiência do Saresp, assim como apontaram os resultados dos estudos de Riani e Rios-Neto (2008) e Franco e Menezes-Filho (2012).

A Tabela 2 traz a distribuição dos alunos da amostra, segundo a faixa de renda familiar, conforme a classificação da proficiência em matemática alcançada. Observa-se que 0 percentual de alunos com renda familiar de até $\mathrm{R} \$ 850$, ou próxima de um salário mínimo, é decrescente à medida que os níveis de proficiência aumentam, isto é, as maiores proporções de alunos nessa faixa de renda pertencem ao nível mais baixo de proficiência, com $16 \%$, enquanto as menores concentram-se no nível AV, aproximadamente $5 \%$. 0 mesmo é verificado para os alunos cuja renda familiar corresponde a quase dois salários mínimos, 
com valores entre $R \$ 851$ e $R$ \$ 1.275: o percentual de alunos no nível $A B$ é mais do que o dobro daquele associado ao nível AV. Para os alunos com proficiência BA e AD em matemática, predominam rendas familiares entre dois e três salários mínimos (de $R \$ 1.276$ a $\mathrm{R} \$ 2.125)$, com $33,50 \%$ e $32,20 \%$, respectivamente.

TABELA 2

Distribuição dos alunos da 3a série do ensino médio da amostra, por níveis de proficiência em matemática, segundo faixas de renda familiar

Estado de São Paulo - 2013

Em porcentagem

Níveis de proficiência em matemática

\begin{tabular}{lcccc} 
Faixas de renda familiar & $\begin{array}{c}\text { Abaixo do básico } \\
\text { (AB) }\end{array}$ & Básico (BA) & Adequado (AD) & Avançado (AV) \\
\hline Até $\mathrm{R} \$$ 850 & 16,04 & 9,71 & 5,70 & 4,89 \\
R\$ 851 a R\$ 1.275 & 31,14 & 25,63 & 17,04 & 13,33 \\
R\$ 1.276 a R\$ 2.125 & 30,33 & 33,50 & 32,20 & 29,44 \\
R\$ 2.126 a R\$ 4.250 & 17,48 & 24,17 & 32,27 & 34,33 \\
Mais de R\$ 4.250 & 5,00 & 6,99 & 12,79 & 18,00 \\
Número de obs. & $\mathbf{9 2 . 9 3 3}$ & $\mathbf{8 7 . 2 9 3}$ & $\mathbf{1 3 . 9 2 7}$ & $\mathbf{9 0 0}$ \\
\hline
\end{tabular}

Fonte: Saresp 2013.

A Tabela 2 mostra, ainda, que as rendas familiares acima de três e próximas ou superiores a seis salários mínimos, indicadas, respectivamente, pelas faixas entre $R \$ 2.126 \mathrm{e}$ $R \$ 4.250$ e acima de $R \$ 4.250$, são observadas com maiores percentuais para os alunos com nível AV de proficiência em matemática. Para as duas faixas de renda mais altas, verifica-se decréscimo no percentual de alunos à medida que o nível de proficiência melhora, exibindo comportamento oposto àquele identificado nas faixas de renda até $\mathrm{R} \$ 850$ e de $\mathrm{R} \$ 851 \mathrm{a}$ $R \$ 2.125$. Enquanto para a faixa de renda de $R \$ 2.126$ a $R \$ 4.250$ os percentuais de alunos variam entre, aproximadamente, $17,5 \%$ e $34 \%$, o percentual de alunos cuja renda familiar é próxima ou superior a seis salários mínimos é quase quatro vezes maior no nível AV de proficiência, quando comparado ao nível $A B$. Tais resultados sugerem que, à medida que a renda familiar aumenta, melhora também o desempenho escolar do aluno, uma vez que faixas de renda mais altas concentram os maiores percentuais de alunos nos níveis AD e AV.

Os resultados obtidos a partir da análise descritiva realizada seguem na mesma direção das conclusões dos estudos de Ferrão et al. (2001), Soares e Mendonça (2003) e Jesus e Laros (2004), confirmando que melhores desempenhos estão associados a rendas familiares mais altas e, portanto, a melhores condições financeiras.

\section{Modelos propostos com variáveis binárias}

Com o objetivo de investigar o impacto de variáveis associadas às características familiares sobre o desempenho dos alunos, no presente estudo, foram utilizados modelos de regressão linear cuja variável resposta, ou explicada, é a proficiência em matemática, representada por Profic_mat, e as variáveis independentes, ou explicativas, são renda 
familiar e escolaridade materna, indicadas, respectivamente, por Renda e Esc_mae. Os modelos propostos são apresentados nas expressões (1), (2) e (3).

Profic_mat ${ }_{i}=\beta_{0}+\beta_{1}$ Renda $_{1}+\varepsilon_{i}$

Profic_mat ${ }_{i}=\beta_{0}+\beta_{2} E s C_{-} m a e_{i}+\varepsilon_{i}$

Profic_mat $_{i}=\beta_{0}+\beta_{1}$ Renda $+\beta_{2}$ Esc_mae $_{i}+\varepsilon_{i}$

Enquanto nos modelos (1) e (2) a proficiência é explicada apenas pelas variáveis renda e escolaridade da mãe, respectivamente, no modelo (3) avalia-se o impacto das duas variáveis, conjuntamente, sobre a proficiência em matemática do aluno da $3^{a}$ série do ensino médio da rede pública.

No modelo (1), $\beta_{0}$ representa a nota em matemática do aluno quando a renda familiar é inferior a $\mathrm{R} \$$ 850. Para o modelo (2), a interpretação do $\beta_{0}$ é baseada nas notas dos alunos cuja mãe não tem estudo. No modelo (3), tal parâmetro está associado ao desempenho dos alunos com renda familiar inferior a $\mathrm{R} \$ 850$ e que, ao mesmo tempo, a mãe não tem qualquer nível de instrução. Em todos os modelos propostos, $\beta_{1}$ e $\beta_{2}$ são os parâmetros da renda familiar e do nível de instrução da mãe, nesta ordem, e mostram a influência destas variáveis sobre a proficiência em matemática. 0 subscrito $i$ indica 0 aluno, $i=1, \ldots, N$, com $N$ representando a quantidade de alunos da amostra elaborada a partir dos dados do Saresp de 2013. Em todos os modelos, $\varepsilon_{i}$ é o termo erro e representa todos os fatores que afetam a proficiência em matemática, além da renda familiar e da escolaridade materna.

Devido ao caráter qualitativo das variáveis renda familiar e escolaridade materna, foram utilizadas variáveis dicotômicas ${ }^{6}$ para incluir tais informações no modelo. Como a questão sobre a renda familiar dispõe de cinco alternativas para respostas, conforme apresentado no Quadro 3, foram necessárias quatro variáveis dicotômicas, definidas conforme a faixa de renda. Assumindo a renda inferior a $\mathrm{R} \$ 850$ como faixa de renda base, ou seja, considerando a renda próxima de um salário mínimo como ponto de referência na comparação com as demais, as variáveis dicotômicas apresentam-se como mostra a expressão (4). Não é necessário definir mais uma variável para representar a última alternativa da questão sobre a renda familiar, uma vez que, ao assumir iguais a zero as quatro variáveis definidas em (4), subentende-se que a referência é a renda familiar inferior a R\$ 850 (TRIOLA, 2008).

\footnotetext{
${ }^{6}$ Também chamadas variáveis binárias ou zero-um, as variáveis dicotômicas auxiliam a introdução de informações qualitativas em modelos de regressão. Tais variáveis assumem apenas dois valores (em geral, 0 e 1) para indicar a presença ou ausência de determinada característica (GUJARATI, 2000; HARDY, 1993).
} 


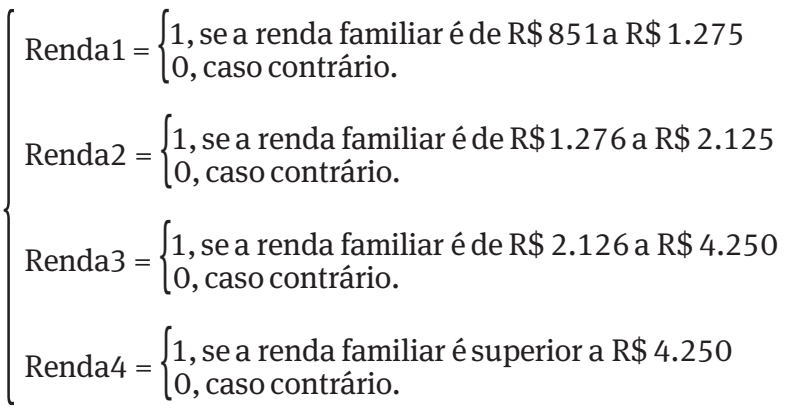

A questão referente ao nível de instrução da mãe do aluno também é qualitativa e possui dez alternativas, como mostrado no Quadro 2. Para que a quantidade de variáveis dicotômicas fosse reduzida, evitando, assim, a obtenção de modelos com um número demasiadamente grande de variáveis, as mães com ensino fundamental incompleto, de $1^{\underline{a}}$ a $4^{\underline{a}}$ série e de $1^{\underline{a}}$ a $8^{\underline{a}}$ série, foram alocadas em um mesmo grupo e aquelas com ensino fundamental completo, de $1^{\underline{a}}$ a $4^{\underline{a}}$ série e de $1^{\underline{a}}$ a $8^{\underline{a}}$ série, em outro grupo. Dessa forma, as variáveis dicotômicas foram criadas como apresenta a expressão (5).

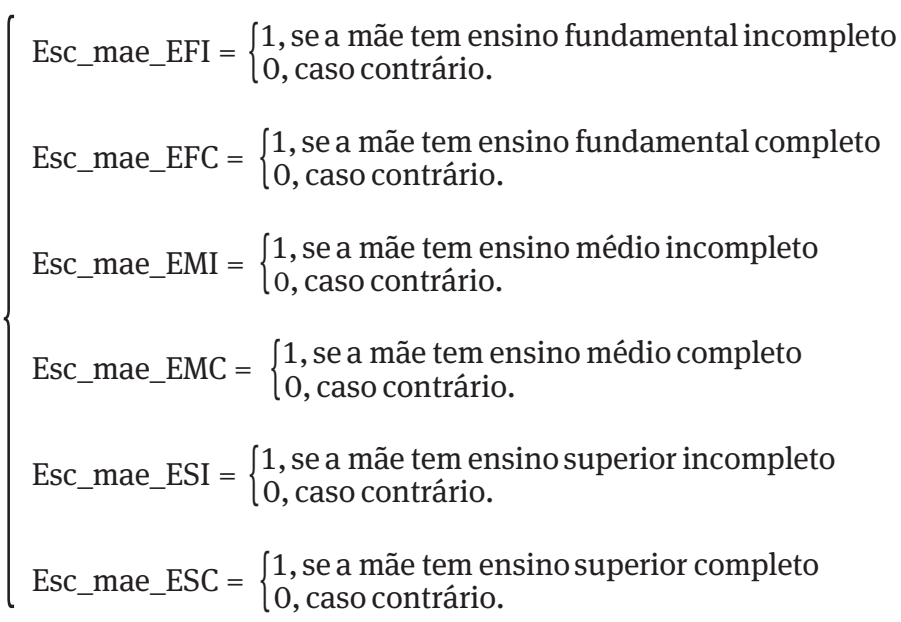

A definição das variáveis dicotômicas possibilitou reescrever os modelos apresentados em (1), (2) e (3) como mostram as expressões (6), (7) e (8).

Profic_mat ${ }_{i}=\beta_{0}+\beta_{11}$ Renda $_{i}+\beta_{12}$ Renda $_{i}+\beta_{13}$ Renda $_{i}+\beta_{14}$ Renda $_{i}+\varepsilon_{i}$

Profic_mat $_{i}=\beta_{0}+\beta_{21} E s c_{-}$mae_EFI $+\beta_{22} E s C_{-}$mae_EFC $i+\beta_{23} E s c_{-}$mae $_{-} E M I_{i}+$

$\beta_{24} E s c_{-} m a e_{-} E M C_{i}+\beta_{25} E s c_{-} m a e_{-} E S I_{i}+\beta_{26} E s C_{-} m a e_{-} E S C_{i}+\varepsilon_{i}$

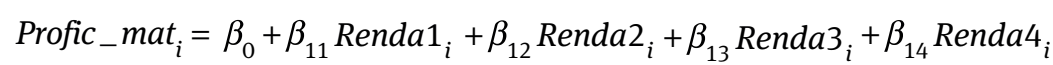

$+\beta_{21} E s c_{-} m a e_{-} E F I_{i}+\beta_{22} E s C_{-} m a e_{-} E F C_{i}+\beta_{23} E s C_{-} m a e_{-} E M I_{i}+$

$+\beta_{24} E s c_{-} m a e_{-} E M C_{i}+\beta_{25} E s c \_m a e \_E S I_{i}+\beta_{26} E s c_{-} m a e \_E S C_{i}+\varepsilon_{i}$ 
No modelo (6), $\beta_{0}$ tem a mesma interpretação dada ao intercepto do modelo (1), ou seja, representa a média em matemática alcançada pelo aluno cuja renda familiar é inferior a $\mathrm{R} \$$ 850. Os demais parâmetros, representados por $\beta_{11}, \beta_{12}, \beta_{13}$ e $\beta_{14}$, mostram, respectivamente, o efeito das faixas de renda definidas em (4). Para o modelo (7), $\beta_{0}$ também representa a média em matemática alcançada pelos alunos cuja mãe não tem estudo, como no modelo (2), e os parâmetros $\beta_{21}, \beta_{22}, \beta_{23}, \beta_{24}, \beta_{25}$ e $\beta_{26}$ correspondem ao acréscimo na nota média do aluno a partir do nível de instrução da mãe, especificados em (5). As mesmas interpretações são válidas para o modelo (8), no qual $\beta_{0}$ representa a média em matemática alcançada pelo aluno cuja mãe não tem estudo e com a mais baixa renda familiar. As interpretações dos parâmetros nos modelos (6), (7) e (8) são baseadas nos valores estimados para $\beta_{0}$.

Interações entre variáveis de renda familiar e de escolaridade da mãe também foram consideradas, porém, em nenhum dos modelos a inclusão de qualquer interação contribuiu para a melhoria dos resultados e, portanto, foram descartadas.

Como critério de seleção de modelos, considerou-se mais adequado aos dados aquele com maior coeficiente de determinação, representado por $R^{2}$, estatística que informa a proporção da variação na variável dependente atribuída a uma ou mais variáveis explicativas (GUJARATI, 2000).

\section{Resultados e discussão}

Utilizand o o software SPSS, estimativas foram obtidas para os parâmetros dos modelos apresentados em (6), (7) e (8), considerando os alunos agrupados conforme o nível de proficiência em matemática. Tal procedimento foi adotado devido às diferenças observadas nas Tabelas 1 e 2, no que se referem à escolaridade da mãe e à renda familiar. A Tabela 1 possibilitou observar que, para mães com ensino fundamental incompleto, o percentual de alunos classificados no nível abaixo do básico é o dobro daquele verificado para proficiência avançada em matemática. Merecem destaque também os percentuais de alunos com proficiência abaixo do básico e avançado em matemática, cujas mães possuem ensino superior completo: enquanto o nível mais alto de proficiência abarcou $25 \%$ dos alunos, no nível abaixo do básico o percentual registrado foi próximo de 5,5\%, quase cinco vezes menor. Na Tabela 2, diferenças significativas também foram observadas para alunos de famílias com rendas de até $R \$ 850$ e acima de $R \$ 4.250$, de acordo com o nível de proficiência em matemática. Enquanto apenas $5 \%$ dos alunos pertencentes ao nível avançado têm renda familiar de até $\mathrm{R} \$ 850$, no nível abaixo do básico tal percentual foi três vezes maior. Ainda observa-se que somente $5 \%$ dos alunos com proficiência em matemática abaixo do básico têm renda familiar superior a $\mathrm{R} \$ 4.250$, enquanto para aqueles com nível avançado tal percentual é quase quatro vezes maior. Diante disso, as estimativas obtidas para os parâmetros das covariáveis associadas à renda familiar e à escolaridade da mãe, ignorando os níveis de proficiência dos alunos, poderiam resultar em valores equivocados e induzir a interpretações inadequadas. 
Concomitantemente, foi calculado o valor do $R^{2}$ associado a cada modelo. Enquanto para os modelos (6) e (7) os valores do $R^{2}$ foram, respectivamente, 0,36 e 0,30, mostrando que $36 \%$ da variabilidade da proficiência em matemática é explicada por variáveis associadas à renda familiar e $30 \%$ por variáveis associadas à escolaridade materna, para o modelo (8) o $R^{2}$ correspondeu a 0,52 , evidenciando que $52 \%$ da variabilidade do desempenho do aluno é explicada pelas variáveis renda e escolaridade da mãe, quando consideradas em um único modelo. Uma vez selecionado o modelo apresentado em (8) como o mais adequado aos dados, conforme maior valor observado para $R^{2}$, as estimativas obtidas são apresentadas na Tabela 3.

Para os alunos do nível $A B$ de proficiência em matemática, a média alcançada por alunos cuja renda familiar é inferior a $\mathrm{R} \$ 850$ e a mãe não estudou foi de 228,38 pontos. Dadas as estimativas positivas e significativas para $\beta_{11}, \beta_{12}, \beta_{13}$ e $\beta_{14}$, os quais indicam rendas entre $R \$ 851$ e $R \$ 1.275, R \$ 1.276$ e $R \$ 2.125, R \$ 2.126$ e $R \$ 4.250$ e superior a $R \$ 4.250$, respectivamente, pode-se verificar que os alunos com qualquer uma das faixas de renda tiveram notas em matemática maiores quando comparadas àquelas obtidas por alunos de mais baixa renda.

TABELA 3

Estimativas para as variáveis associadas à renda familiar e escolaridade da mãe associadas às proficiências em matemática dos alunos da 3 a série do ensino médio da amostra

Estado de São Paulo - 2013

\begin{tabular}{|c|c|c|c|c|}
\hline \multirow{2}{*}{$\begin{array}{l}\text { Parâmetros associados à renda } \\
\text { familiar e escolaridade da mãe }\end{array}$} & \multicolumn{4}{|c|}{ Níveis de proficiência em matemática } \\
\hline & $\begin{array}{c}\text { Abaixo do básico } \\
\text { (AB) }\end{array}$ & Básico (BA) & Adequado (AD) & $\begin{array}{l}\text { Avançado } \\
\text { (AV) }\end{array}$ \\
\hline $\begin{array}{l}\text { Renda inferior a } \mathrm{R} \$ 850 \text { e sem } \\
\text { estudo }\left(\beta_{0}\right)\end{array}$ & $228,38^{\star \star \star}(0,536)$ & $300,46^{\star \star \star}(0,540)$ & $364,83(1,059)$ & $408,83(3,383)$ \\
\hline $\mathrm{R} \$ 851$ a $\mathrm{R} \$ 1.275\left(\beta_{11}\right)$ & $2,937^{\star \star \star}(0,281)$ & $1,182^{\star \star \star}(0,261)$ & $0,241(0,520)$ & $-0,376(1,232)$ \\
\hline $\mathrm{R} \$ 1.276$ a $\mathrm{R} \$ 2.125\left(\beta_{12}\right)$ & $4,750^{\star \star \star}(0,284)$ & $3,109^{\star \star \star}(0,254)$ & $0,734(0,490)$ & $0,423(1,153)$ \\
\hline $\mathrm{R} \$ 2.126$ a $\mathrm{R} \$ 4.250\left(\beta_{13}\right)$ & $5,103^{\star \star \star}(0,321)$ & $4,583^{\star \star \star}(0,267)$ & $1,816^{\star \star \star}(0,495)$ & $0,394(1,156)$ \\
\hline Mais de $R \$ 4.250\left(\beta_{14}\right)$ & $3,799^{\star \star \star}(0,476)$ & $5,086^{\star \star \star}(0,351)$ & $2,392^{\star \star \star}(0,561)$ & $0,191(1,237)$ \\
\hline $\begin{array}{l}\text { Ensino fundamental incompleto } \\
\left(\beta_{21}\right)\end{array}$ & $4,120^{\star \star \star}(0,536)$ & $2,131^{\star \star \star}(0,527)$ & $0,391(1,028)$ & $-0,966(3,262)$ \\
\hline Ensino fundamental completo $\left(\beta_{22}\right)$ & $4,128^{\star \star \star}(0,541)$ & $2,519^{\star \star \star}(0,530)$ & $0,334(1,028)$ & $-0,638(3,256)$ \\
\hline Ensino médio incompleto $\left(\beta_{23}\right)$ & $5,403^{\star \star \star}(0,611)$ & $3,616^{\star \star \star}(0,565)$ & $1,008(1,066)$ & $1,577(3,319)$ \\
\hline Ensino médio completo $\left(\beta_{24}\right)$ & $5,865^{\star \star \star}(0,541)$ & $4,583^{\star \star \star}(0,524)$ & $1,204(1,012)$ & $-0,509(3,223)$ \\
\hline Ensino superior incompleto $\left(\beta_{25}\right)$ & $4,464^{\star \star \star}(0,715)$ & $5,902^{\star \star \star}(0,606)$ & $1,735(1,090)$ & $0,083(3,341)$ \\
\hline Ensino superior completo $\left(\beta_{26}\right)$ & $4,020^{\star \star \star}(0,651)$ & $7,248^{\star \star \star}(0,565)$ & $2,281^{\star \star \star}(1,036)$ & $-0,158(3,235)$ \\
\hline
\end{tabular}

Fonte: Saresp 2013 - Questionário socioeconômico.

Nota: Os valores entre parênteses indicam o desvio padrão associado a cada estimativa.

*** Indica que a estimativa é significativa ao nível de 1\% (GELMAN et al., 2009).

Pode-se afirmar ainda que, conforme cresceu a renda familiar, a nota também aumentou. Porém, tal comportamento é observado somente até a renda familiar de R\$2.126 a $R \$ 4.250$. Enquanto os acréscimos às médias dos alunos com rendas familiares de $R \$ 851$ a $R \$ 1.275$, de $R \$ 1.276$ a $R \$ 2.125$ e de $R \$ 2.126$ a $R \$ 4.250$ foram crescentes e iguais 
a $2,937,4,750$ e 5,103, nesta ordem, alunos cuja renda familiar é superior a $\mathrm{R} \$ 4.250$ tiveram um acréscimo de 3,799, ou seja, o comportamento crescente não se mantém entre as estimativas destes parâmetros. Caso fosse mantida, a estimativa para $\beta_{14}$, associado à renda familiar superior a $\mathrm{R} \$ 4.250$, seria a maior quando comparada às estimativas de $\beta_{11}$, $\beta_{12}$ e $\beta_{13}$, os quais representam os parâmetros das rendas familiares inferiores.

Ainda para os alunos pertencentes ao nível $A B$ de proficiência em matemática, as estimativas mostram que os anos de estudo da mãe foram importantes para seus desempenhos no Saresp 2013, pois as estimativas obtidas para os parâmetros $\beta_{21}, \beta_{22}, \beta_{23}, \beta_{24}, \beta_{25}$ e $\beta_{26}$, correspondentes aos níveis de escolaridade fundamental incompleto, fundamental completo, médio incompleto, médio completo, superior incompleto e superior completo, respectivamente, foram todas significativas. No entanto, embora crescentes, tal ordem se manteve somente até o nível de escolaridade correspondente ao ensino médio completo. Embora significativas, as estimativas associadas aos níveis de instrução ensino superior incompleto e completo indicam acréscimos menores na nota do aluno, quando comparados àqueles identificados para o ensino médio incompleto e completo. Dessa foram, conclui-se que as maiores contribuições foram proporcionadas pelas mães com ensino médio completo e incompleto, quando comparadas com os demais níveis de escolaridade.

Considerando os alunos do nível BA de proficiência em matemática, observa-se que notas maiores foram obtidas à medida que a renda familiar aumenta, já que todas as estimativas para $\beta_{11}, \beta_{12}, \beta_{13}$ e $\beta_{14}$ foram significativas e crescentes, sugerindo que rendas familiares mais altas resultam em melhores desempenhos em matemática. Ainda para os alunos classificados no nível BA pelo Saresp de 2013, observa-se que a escolaridade da mãe atuou positivamente sobre o desempenho do filho, uma vez que níveis mais altos de instrução materna proporcionaram maiores acréscimos à média do aluno.

Enquanto a nota dos alunos classificados no nível AD de proficiência em matemática aumentou para as rendas superiores a $\mathrm{R} \$ 2.126$, quando comparadas com a média próxima de 365 , alcançada por alunos com renda inferior a $R \$ 850$ e mães sem estudo, rendas entre $\mathrm{R} \$ 851$ e R\$2.125 não tiveram efeitos sobre o desempenho dos alunos pertencentes a esse nível de proficiência, já que as estimativas para $\beta_{11}$ e $\beta_{12}$ não foram significativas. No que se refere ao nível de instrução da mãe, pode-se verificar, a partir dos valores mostrados na Tabela 3, que tal característica não influenciou o desempenho dos alunos classificados no nível $A D$ de proficiência, a não ser que a mãe tenha o ensino superior completo, como mostra a significância da estimativa para $\beta_{26}$.

Para os alunos que se classificaram no nível AV, cuja média foi de 408,8 para aqueles com renda familiar inferior a $\mathrm{R} \$ 850,00$ e cuja mãe não tem estudo, não se mostrou relevante, para o desempenho, se a família tem renda inferior a $R \$ 850$, entre $R \$ 851$ e $R \$ 4.250$ ou superior a $\mathrm{R} \$ 4.250$, já que as estimativas obtidas para os parâmetros $\beta_{11}, \beta_{12}, \beta_{13}$ e $\beta_{14}$ não foram significativas. 0 nível de instrução materno também não influenciou o desempenho destes alunos, pois nenhum parâmetro associado a tal característica foi significativo. 
Barros e Mendonça (1997) encontraram evidências de que o desempenho do aluno está sob maior influência da escolaridade dos pais do que da renda familiar. A partir dos resultados obtidos na presente pesquisa empírica, não se pode fazer tal afirmação, uma vez que, para os alunos pertencentes aos níveis abaixo do básico e básico, as contribuições associadas à renda familiar e escolaridade da mãe foram semelhantes, conforme valores mostrados na Tabela 3. Já para os alunos do nível avançado de proficiência, nem a renda familiar nem a escolaridade materna contribuíram para um melhor desempenho escolar do indivíduo.

A interpretação das estimativas obtidas para os parâmetros dos modelos propostos permite afirmar que os resultados alcançados no presente estudo corroboram as conclusões de Ferrão et al. (2001) e Alves e Soares (2008), sobre fatores associados à família que impactam o desempenho do aluno. A renda familiar e a escolaridade da mãe demonstraram ser variáveis importantes no que se refere a uma educação de qualidade, visto o impacto positivo sobre a proficiência em matemática. No entanto, diferentemente dos demais trabalhos que investigaram os efeitos destas variáveis no processo de escolarização, a presente pesquisa identificou que, embora a renda familiar seja um fator extraescolar significativo para o desempenho do aluno, este resultado não deve ser generalizado para todos os níveis de proficiência em matemática. Adicionalmente, os resultados evidenciaram a importância da educação materna nos indicadores educacionais, porém, somente até o nível básico de proficiência. A ausência de estimativas significativas impossibilitou a generalização do resultado encontrado por Franco e Menezes-Filho (2012), que destacaram a relevância da educação da mãe sobre a trajetória escolar do filho.

A renda familiar e a escolaridade da mãe demonstraram que existe uma estratificação educacional no Brasil, pois a trajetória escolar do aluno está bastante relacionada com a sua origem social. Em termos de políticas públicas, é importante conhecer os fatores que podem minimizar a importância da origem social do aluno, buscando tornar o sistema mais igualitário.

\section{Conclusão}

Identificar os fatores que atuam sobre a melhoria do ensino e em que medida esses fatores exercem influência é importante, visto que os retornos do aumento da escolaridade podem contribuir não só para o crescimento da renda futura do indivíduo, mas, principalmente, para o desenvolvimento econômico do país. Nesse sentido, o presente estudo propôs-se a investigar os efeitos de fatores extraescolares, especificamente a renda familiar e a escolaridade materna, sobre o desempenho escolar do aluno. Para tanto, foram utilizados dados sobre o desempenho em matemática e sobre as características familiares dos alunos da $3^{a}$ série do ensino médio da rede pública estadual de ensino, captados pelo Saresp em 2013, disponibilizados pela Secretaria Estadual de Educação (SEE/SP).

A análise empírica realizada nesta pesquisa mostrou que a atuação do nível socioeconômico, medido pela renda familiar, ocorre de maneira controversa dependendo do nível de 
proficiência em que o aluno se encontra. Enquanto nos níveis abaixo do básico e básico a influência aumentou até a faixa de renda entre $R \$ 2.126$ e $R$ \$ 4.250, para os níveis adequado e avançado, a renda familiar não impactou o desempenho escolar do aluno. Similarmente, a escolaridade da mãe atuou positivamente somente sobre o desempenho dos alunos com níveis de proficiência abaixo do básico e básico, sendo irrelevante para os demais.

Os resultados alcançados, a partir das estimativas obtidas para os parâmetros do modelo proposto, impedem afirmar que rendas familiares mais altas e mães com níveis mais altos de instrução proporcionaram melhores resultados no Saresp de 2103. Tais afirmações levam à reflexão acerca da existência e identificação de um limite de atuação, tanto do nível socioeconômico familiar quanto da escolaridade materna, sobre o desempenho escolar do aluno.

As estimativas obtidas utilizand o o software SPSS possibilitaram verificar que, embora a renda caracterize um fator que impacta o desempenho do aluno, a influência das rendas mais altas ou mais baixas não é constante sobre as médias em matemática, conforme o nível de proficiência. 0 mesmo pode ser argumentado no que se refere à escolaridade materna. Nesse sentido, ainda que a conclusão desta pesquisa empírica corrobore os resultados de autores como Soares e Mendonça (2003), Alves e Soares (2008), os quais afirmam que a condição socioeconômica do aluno influencia o seu desempenho, não se pode afirmar que rendas familiares mais altas e mães com níveis de instrução mais elevados resultam em melhores desempenhos escolares. Tal fato indica a necessidade de um refinamento analítico no sentido de responder como determinantes extraescolares associados à família favorecem ou interferem no desempenho dos alunos e por quais caminhos isso se realiza. A continuidade desta pesquisa se torna fundamental para a inclusão de outras variáveis relacionadas à condição socioeconômica no modelo e, assim, estender a compreensão sobre os elementos que interferem e/ou favorecem um melhor desempenho na escolarização básica.

\section{Referências}

ALVES, M. T. G.; SOARES, J. F. O efeito das escolas no aprendizado dos alunos: um estudo com dados longitudinais no ensino fundamental. Educação e Pesquisa, São Paulo, v. 34, n. 3, p. 527-544, set./dez. 2008.

BARBOSA, M. E. F.; FERNANDES, C. A escola brasileira faz diferença? Uma investigação dos efeitos da escola na proficiência em matemática dos alunos da 4a série. In: CRESO, F. (org.). Avaliação, ciclos e promoção na educação. Porto Alegre: Artmed Editora, 2001. p. 155-172.

BARROS, R.; MENDONÇA, R. 0 impacto de gestão escolar sobre o desempenho educacional. Research Department Publications 3000, Inter-American Development Bank, Research Department, 1997. Disponivel em: 〈http://www.iadb.org/res/publications/pubfiles/pubR-301. pdf〉. Acesso em: 28 set. 2016.

COLEMAN, J.; CAMPBELL, E.; HOBSON, C.; MCPARTLAND, J.; MOOD, A.; WEINFELD, F.; YORK, R. Equality of educational opportunity. Washington, DC: National Center for Educational Statistics, 1966. Disponível em: 〈http://files.eric.ed.gov/fulltext/ED012275.pdf〉. Acesso em: 30 set. 2016. 
FERRÃO, M. E.; BELTRÃO, K. I.; FERNANDES, C.; SANTOS, D.; SUÁREZ, M.; ANDRADE, A. do C. O Saeb - Sistema Nacional de Avaliação da Educação Básica: objetivos, características e contribuições na investigação da escola eficaz. Revista Brasileira de Estudos de População, v. 18, n. 1/2, p. 111-130, jan./dez. 2001.

FRANCO, A. M. P.; MENEZES FILHO, N. Uma análise de rankings de escolas brasileiras com dados do SAEB. Estudos Econômicos, São Paulo, v. 42, n. 2, p. 263-283, abr./jun. 2012.

GELMAN, A.; CARLIN, J. B.; STERN, H. S.; RUBIN, D. B. Bayesian data analysis. 2. ed. Washington, DC: Chapman \& Hall, 2009.

GUJARATI, D. Econometria básica. 3. ed. São Paulo: Makron Boooks, 2000.

HARDY, M. A. Regression with dummy variables. Newbury Park: Sage Publications, 1993.

JESUS, G. R. de; LAROS, J. A. Eficácia escolar: regressão multinível com dados de avaliação em larga escala. Avaliação Psicológica, Campinas, v. 3, n. 2, p. 93-106, nov. 2004.

PALERMO, G.; SILVA, D. B. do N.; NOVELLINO, M. S. F. Fatores associados ao desempenho escolar: uma análise da proficiência em matemática dos alunos do $5^{\circ}$ ano do ensino fundamental da rede municipal do Rio de Janeiro. Revista Brasileira de Estudos de População, Rio de Janeiro, v. 31, n. 2, p. 367-394, jul./dez. 2014.

RIANI, J. de L. R.; RIOS-NETO, E. L. G. Background familiar versus perfil escolar do município: qual possui maior impacto no resultado educacional dos alunos brasileiros? Revista Brasileira de Estudos de População, São Paulo, v. 25, n. 2, p. 251-269, jul./dez. 2008.

RIOS-NETO, E. L. G.; CÉSAR, C. C.; RIANI, J. de L. R. Estratificação educacional e progressão escolar por série no Brasil. Pesquisa e Planejamento Econômico, Rio de Janeiro, v. 32, n. 3, p. 395-415, dez. 2002.

SÃO PAULO (Estado). Secretaria da Educação. Fundação para o Desenvolvimento da Educação - FRD. Relatório pedagógico SARESP 2013 - Matemática. São Paulo: Secretaria da Educação do Estado de São Paulo, 2013.

SOARES, J. F.; COLLARES, A. C. M. Recursos familiares e o desempenho cognitivo dos alunos do ensino básico brasileiro. Dados: Revista de Ciências Sociais, Rio de Janeiro, v. 49, n. 3, p. 615-650, 2006.

SOARES, J. F.; CÉSAR, C. C.; MAMBRINI, J. Determinantes de desempenho dos alunos do ensino básico brasileiro: evidências do SAEB de 1997. In: CRESO, F. (org.). Avaliação, ciclos e promoção na educação. Porto Alegre: Artmed Editora, 2001. p. 121-153.

SOARES, T. M.; MENDONÇA, M. C. M. Construção de um modelo de regressão hierárquico para os dados do Simave-2000. Pesquisa Operacional, v. 23, n. 3, p. 421-441, set./dez. 2003.

TRIOLA, M. F. Introdução à estatística. 10. ed. Rio de Janeiro: LTC, 2008.

\section{Sobre a autora}

Camila Fernanda Bassetto é doutora em Ciências pelo Programa de Engenharia de Produção, da Escola de Engenharia de São Carlos (EESC), da Universidade de São Paulo (USP) e mestre em estatística pela Universidade Federal de São Carlos (UFSCar). Professora do departamento de Ciências da Educação na Faculdade de Ciências e Letras (FCL), da Universidade Estadual Paulista “Júlio de Mesquita Filho" (Unesp), campus de Araraquara. 


\title{
Endereço para correspondência
}

Faculdade de Ciências e Letras - FCL/Unesp Departamento de Ciências da Educação Rod. Araraquara-Jaú Km 1 - Machados

14800-901 - Araraquara-SP, Brasil

\begin{abstract}
Family background and school performance: an approach with binary variables from SARESP results

This study researches the impact of outside school factors on individuals' schooling. Income and maternal schooling are taken into consideration when measuring the effect of familiar aspects on students' performance in Mathematics. Therefore, we used data referring to the Mathematics grade of students in $3^{\text {rd }}$ year High School, and the answers provided by their parents for the socioeconomic questionnaire, applied by SARESP in its 2013 edition. Models with binary variables are proposed and estimates for parameters are obtained with SPSS. Results show that students from families with higher incomes indicate better educational performance, but there are restrictions. The level of maternal education positively influences student's performance, but is limited to appropriate proficiency levels.
\end{abstract}

Keywords: Proficiency in mathematics. Family income. Maternal education. SARESP. Binary variables.

\section{Resumen}

Fondo familiar y desempeño escolar: Un enfoque con variables binarias a partir de los resultados del SARESP

El presente estudio investiga el impacto de los factores externos a la escuela sobre la escolarización del individuo. Se consideran la renta y la escolaridad materna para medir el efecto de los aspectos familiares en el desempeño del alumno en Matemáticas. Para eso, se utilzaron datos sobre la nota en Matemáticas de los alumnos de la tercera serie de Enseñanza Media y las respuestas de los padres al cuestionario socioeconómico aplicado por la Secretaria da Educação do Estado de São Paulo (SARESP) en la edición de 2013. Se proponen modelos con variables binarias y estimativos para los parámetros que se obtienen con el SPSS. Los resultados muestran que las rentas más altas proporcionan mejores resultados educativos, a pesar de lo cual hay restricciones: el nivel de escolaridad materna actúa positivamente sobre el desempeño del alumno, pero está limitado al nivel adecuado de competencia.

Palabras clave: Competencia en matemáticas. Fondo familiar. Escolaridad materna. SARESP. Variables binarias. 